Los Alamos archives: gr-qc/9408039

(C) H.C. Rosu (1998); MPL-A 13, 695-699 (1998)

\title{
ON THE REMOTE SENSING OF HAWKING GREY PULSES
}

\author{
HARET C. ROSU \\ Instituto de Física, Universidad de Guanajuato, Apdo Postal E-143, León, Gto, Mexico
}

\begin{abstract}
This is a short note on the black hole remote-sensing problem, i.e., finding out 'surface' temperature distributions of various types of small (micron-sized) black holes from the spectral measurements of their Hawking grey pulses. Chen's modified Moebius inverse transform is illustrated in this context.
\end{abstract}

The grey body radiation spectrum from various types of black holes is an interesting topic, both at the fundamental level ${ }^{1}$ and at the experimental (astrophysical) one. In a previous paper, ${ }^{2}$ I have applied the modified Moebius transform (henceforth MMT) due to Chen ${ }^{3}$ to the so-called distorted black holes, a terminology used by Geroch and Hartle ${ }^{4}$ to denote axially symmetric black hole Einstein solutions generated by Weyl's technique ${ }^{5}$ from flat space Laplace equation. Here I provide some further details.

In an astrophysical context, one expects quite naturally to encounter black holes carrying on an "external" distribution of matter. Examples are as follows

(i) Weyl black holes, to which also Schwarzschild black holes belong. As is well-known the metric for a static axially symmetric spacetime in Weyl's canonical coordinates $(t, R, z, \phi$,$) reads$

$$
d s^{2}=-e^{\phi} d t^{2}+e^{\nu-\phi}\left(d R^{2}+d z^{2}\right)+R^{2} e^{-\phi} d \phi^{2},
$$

where the metric potentials $\phi$ and $\nu$ are functions of the coordinates $R$ and $z$ only. The $\phi$ potential is at the same time a solution of the polar Laplace equation, a remarkable fact discovered by Weyl, while the $\nu$ potential is easily obtainable from the first one in the form of an integral. ${ }^{6}$ The astrophysical systems to which these Weyl black holes are mostly associated, in an already extensive literature, ${ }^{7}$ are giant black hole- disk (either thin or thick) configurations which are thought to model active galactic nuclei (AGN). These giant systems will not be included further in our considerations but one is free to think of such systems scaled down by many orders of magnitude.

(ii) Black holes with Einstein shells (again either thick or thin) which are spherically symmetric, static objects constructed of test particles travelling along closed geodesics around black holes. They were first considered by Einstein, ${ }^{8}$ who introduced a well-known ansatz for their energy-momentum tensor.

(iii) Primordial black holes (PBH) and/or any mini black hole hovering through the universe and carrying on some matter distributions. As we already stated, one 
may think of the first two models scaled down by many orders of magnitude, or of any other conceivable matter distribution accreated around such small black holes.

(iv) Dirty black holes in the sense of Visser, ${ }^{9}$ i.e., black holes in interaction with various classical fields, for which the Hawking temperature appears to be supressed relative to the vacuum black holes of equal area.

In the following I shall use the notion of 'distorted black holes' for all these non-isolated black holes. In some cases, the external distribution of matter can be of such a kind as to disturb only slightly the pure horizon Hawking radiation and consequently the character of the problem as an inverse grey-body radiation problem is preserved. I recall that Hawking radiation by itself is seriously distorted from a pure black-body radiation, especially in the low frequency regime due to a grey-body factor usually identified with the square of the absorption amplitude for the mode. ${ }^{10}$ Thus, the least our remote sensing arguments require is the common thermal description of pure black holes, and it is only for astrophysical reasons that we expect some matter shells (rings, disks, shells, and so on) to occur.

The inverse grey-body problem is a classic one in the field of remote sensing. ${ }^{11}$ Mathematically, it is usually unstable for most numerical implementations, i.e., it is an ill-posed inverse problem. ${ }^{12}$ Among the various procedures to solve it (at least in principle), MMT is a quite unconventional technique, although it belongs to the Laplace transform methods.

Referring to the Planck law we shall notice that it actually is the analytical formula for the power spectrum, which in laboratory physics is also called spectral brightness, or spectral radiance of the black body radiation. The latter notion is used in radiometry to characterize the source spectral properties as a function of position and direction, which at the present time are not known experimentally for black holes. For point, i.e., far away, grey sources the total radiated power spectrum, also called radiant spectral intensity is

$$
W(\nu) \sim \int_{0}^{\infty} A(T) B(\nu, T) d T
$$

where $A(T)$ is the area temperature distribution of the grey body, and $B(\nu, T)$ is the Boltzmann-Planck occupation factor. The inverse grey-body problem is to solve the integral equation for $A(T)$ for given total radiated power spectrum, which may be known either experimentally or within some theoretical model. This problem was solved in principle by Bojarski ${ }^{13}$ by means of a thermodynamic coldness parameter $u=h / k T$, and an area coldness distribution $a(u)$, as more convenient variables to get an inverse Laplace transform of the total radiated power. The coldness distribution is obtained as an expansion in this Laplace transform. More precisely, the total grey power spectrum is rewritten as

$$
W(\nu)=\frac{2 h \nu^{3}}{c^{2}} \int_{0}^{\infty} \frac{a(u)}{\exp (u \nu)-1} d u
$$


and furthermore as

$$
W(\nu)=\frac{2 h \nu^{3}}{c^{2}} \int_{0}^{\infty} \exp (-u \nu) \sum_{n=1}^{\infty}(1 / n) a(u / n) d u .
$$

Therefore the sum under the integral that we shall denote as $f(u)$ is nothing else but the Laplace transform of $g(\nu)=\frac{c^{2}}{2 h \nu^{3}} W(\nu)$.

From the Laplace transform $f(u)$ of a grey body, Chen obtained $a(u)$ by means of the MMT in the form

$$
a(u)=\sum_{n=1}^{\infty} \frac{\mu(n)}{n} f(u / n) .
$$

The Moebius expansion refers to special sums over prime factor-divisors, (dsums) of any number theoretic function $f(n)$, running over all the prime factors of $n, 1$ and $n$ included

$$
f_{1}=\sum_{d \mid n}^{n} f_{2}(d) .
$$

The remarkable fact in this case is that the last term of the sum can be written in turn as sums of $f_{1}$ functions, which are called inverse Moebius transforms (or Moebius d-sums)

$$
f_{2}(n)=\sum_{d \mid n}^{n} \mu(d) f_{1}(n / d)
$$

in which $f_{1}(n)$, i.e., the d-sum, becomes the first term of the Moebius d-sum, and where $\mu(d)$ is the famous Moebius function. Since each $f_{1}$ term in the second expansion is a d-sum, there is clearly an overcounting, unless the Moebius functions are sometimes either naught or negative. The partition of the prime factors of $n$ implied by the Moebius function is such that, by definition, $\mu(1)$ is $1, \mu(n)$ is $(-1)^{r}$ if $n$ includes $r$ distinct prime factors, and $\mu(n)$ is naught in all the other cases. In particular, all the squares have no contribution to the inverse Moebius transforms. That is why the integers selected by the Moebius function are also called square-free integers.

Chen's MMT means to apply such an inversion of finite sums to infinite summations, and to ordinary functions of real continuous variable(s). MMT means that if

$$
f_{1}(x)=\sum_{n=1}^{\infty} f_{2}(x / n)
$$

then

$$
f_{2}(x)=\sum_{n=1}^{\infty} \mu(n) f_{1}(x / n)
$$

For the inverse grey-body problem, $f_{1}(x)=u f(u)$ and $f_{2}(x)=u a(u)$. So, one can get the coldness distribution by multiplying the Laplace transform of the total power spectrum by the coldness parameter, and then applying the MMT. 
I provide now a simple example of the way how the MMT technique works. For micron-sized Schwarzschild black holes $\left(M \sim 10^{24} \mathrm{~g}\right.$ ), no known massive particles are thermally emitted, and according to the calculations of $\mathrm{Page}^{14}$ about $16 \%$ of the Hawking flux goes into photons, the rest being neutrino emission. Let us consider these black holes as grey objects, either by their own, ${ }^{10}$ or even when possessing some matter distribution close to their horizons. The coldness parameter will be in the first case $u_{S}=\frac{1}{\nu} \ln \left(1+\frac{e^{\beta_{h} \hbar \omega}-1}{\Gamma(\omega)}\right)$, where $\beta_{h}$ is the horizon inverse temperature parameter, and $\Gamma(\omega)$ is the penetration factor of the curvature and angular momentum barrier around the black hole, as can be infered from the work of Bekenstein ${ }^{10}$, whereas in the latter case $u_{S}=h / k T_{d}$, where $T_{d}$ can be considered as an effective horizon temperature of the distorted black holes $T_{d}=(8 \pi M)^{-1} \exp (2 \mathcal{U})$, with $\mathcal{U}=1 / 2(1 / 2 \nu-\phi)-\ln (1 / 2 \sqrt{(} R / M){ }^{4}$ Therefore, the coldness distribution will be

$$
a\left(u_{S}\right)=\frac{c^{2}}{2 h \nu^{3}} \sum_{n=1}^{\infty} \frac{\mu(n)}{n} f\left(u_{S} / n\right),
$$

where $f$ is the inverse Laplace transform of the total photon power spectrum.

\section{Acknowledgements}

This work was partially supported by the CONACyT Project 4868-E9406.

\section{References}

1. M. Schiffer, Gen. Rel. Grav. (1995) 27 1, and references therein.

2. H. Rosu, Nuovo Cimento B108 (1993) 1333.

3. N.-x. Chen, Phys. Rev. Lett. 64 (1990) 1193.

4. R. Geroch and J. Hartle, J. Math. Phys. 23 (1982) 680.

5. H. Weyl, Ann. Phys. 54 (1917) 117.

6. J.L. Synge, Relativity: The General Theory (North Holland, Amsterdam, 1964) p. 312.

7. J.P.S. Lemos and P.S. Letelier, Phys. Rev. D49 (1994) 5135, and references therein.

8. A. Einstein, Ann. Math. 40 (1939) 922. For recent papers see P.R. Brady, J. Louko, and E. Poisson, Phys. Rev. D44 (1992) 1891; G.L. Comer, D. Langlois, and P. Peter, Class. Quantum Grav. 10 (1993) L127; G.L. Comer and J. Katz, Class. Quantum Grav. 10 (1993) 1751.

9. M. Visser, Phys. Rev. D46 (1992) 2445.

10. J.D. Bekenstein, Phys. Rev. Lett. 70 (1993) 3680; C.F.E. Holzhey and F. Wilczek, Nucl. Phys. B380 (1992) 447; V. Balasubramanian and F. Larsen, Nucl. Phys. B495 (1997) 206; J.M. Maldacena and A. Stominger, Phys. Rev. D55 (1997) 861.

11. For a concise review, see A. Lakhtakia, Mod. Phys. Lett. B5 (1991) 491.

12. Xin Tan et al, J. Opt. Soc. Am. A11 (1994) 1068.

13. N.N. Bojarski, IEEE Trans. Antennas Propag. 30 (1982) 778.

14. D.N. Page, Phys. Rev. D13 (1976) 198. 